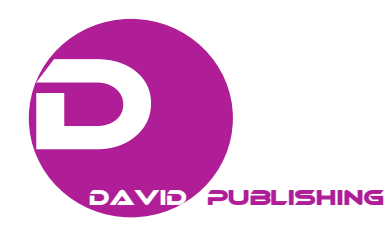

\title{
Parameter Variations of Identified Model for Static Deformation Analysis of Arch Dam
}

\author{
Jiann-Shiun Lew \\ Center of Excellence in Information Systems, Tennessee State University, Tennessee 37209, USA
}

\begin{abstract}
The structural health monitoring of a dam is important for maintaining the safe operation and longevity of the dam system. The structural health of a large dam can be monitored from the measured static deformation. This paper presents an investigation of the parameter variations of the identified model of the measured long-term static deformation for the structural health monitoring of Fui-Tsui Dam, which is located in a very active seismic zone of Taiwan. The measured static deformation is characterized as a function of the measured physical parameters, including the effects of hydrostatic pressure and temperature variation. The identified parameters, associated with the effects of hydrostatic pressure and temperature variation, change with environmental factors, such as flooding, earthquake and foundation change.
\end{abstract}

Key words: Static deformation, large dam, modeling, parameter variation, structural health monitoring.

\section{Introduction}

Large dams are exposed to environmental, geomechanical and hydraulic factors, such as flooding, earthquake, water level, temperature and foundation change. All of these affect structural behavior. Therefore it is a challenging task to develop a reliable SHM (structural health monitoring) system for large dams.

The structural health monitoring of civil infrastructures, such as bridges and dams, is important for the safe operation, hazard prevention, and the longevity of the system $[1,2]$. Significant developments in SHM of civil structures have originated from major construction projects, such as large dams and long-span cable-supported bridges. Regular inspection of dams became a legal requirement in the UK after the failure of a $30-\mathrm{m}$ embankment dam claimed the lives of 254 people near Sheffield, UK in 1864 [3]. The traditional procedure for the evaluation of the structural health condition of dams is through visual inspection. However, visual

Corresponding author: Jiann-Shiun Lew, Ph.D., research professor, research fields: structural health monitoring, system identification and robust control design. inspections have some shortcomings such as significant labor demands and inaccessible critical locations. The problems of visual inspections can be addressed with field tests, which are conducted to monitor the dynamic and static characteristics of structures [4-6]. For a large dam, it is a challenging task to excite structural vibration. Significant vibration measurement data are available only when earthquakes occur. Thus, the structural health of the dam cannot be continuously monitored based on the variation of structural vibration. The use of static deformation measurement is an alternative way to continuously monitor the structural health of a dam. Various methods, based on dynamic and static tests, have been developed to address structural health monitoring and damage identification [4]. Uncertainty in SHM systems, such as environmental and operational variations, may significantly affect the measurements and reduce the reliability of damage identification with false indications of damage $[7,8]$. Therefore, it is necessary to develop robust and reliable approaches to identify accurately damage status and provide an early warning threshold before a disaster occurs. 
Various modeling techniques have been developed that use the measured long-term static deformation for structural health monitoring of dams. A concrete dam is subjected to hydrostatic load and thermal model. The statistical and identification procedures $[9,10]$ have been widely applied to model long-term deformation as a function of hydrostatic load, which is expressed as the polynomials of water level, and thermal model, which is expressed as a sinusoidal function of seasonal temperature effect. A linear least-squares technique is applied to minimize the difference between the measured static deformation and the identified model to get a linear statistical model. Recently, a nonlinear modeling technique [11] was developed for the measured static deformation of Fei-Tsui Dam (Fig. 1), which is located in Taiwan. The results demonstrate that the identified nonlinear model well represents the measured static deformation. This identified model also gives a precise prediction of the future long-term static deformation for structural health monitoring of Fei-Tsui Dam. The results of the previous study demonstrate that the identified parameters of the nonlinear model change with environmental factors, such as flooding and drought. This paper presents a thorough investigation of the identified parameter variations based on a modified approach of this nonlinear modeling technique.

\section{Measurement of Fei-Tsai Dam}

Fei-Tsui Dam (Fig. 1) is $122.5 \mathrm{~m}$ high and the dam crest is $510 \mathrm{~m}$ in length. It is designed with a double curvature arch along the height of the dam. The capacity of this reservoir is about 400 million $\mathrm{m}^{3}$. Since this dam is located in a very active seismic zone of Taiwan, both dynamic and static monitoring systems of the dam are deployed in this structure [6]. There are several static measurement systems in this Feu-Tsui Dam. One of the important static measurement systems is the monitoring of dam deformation. There are five measurement points along the dam height (at levels $172.5 \mathrm{~m}, 150 \mathrm{~m}, 115 \mathrm{~m}$, $90 \mathrm{~m}$ and $57.5 \mathrm{~m}$ ) to measure the radial deformation (Y direction) of the dam [6]. The daily dam deformations and water level were measured at a fixed time in the morning. These static deformation measurements were collected automatically with the use of the laser beams starting from January 1, 1987, and they were recorded to the unit of $0.1 \mathrm{~mm}$ with the initial deformation set as zero. The daily maximum and minimum temperatures at the dam site were also measured. The data were not recorded during dam maintenance time, so the data were unavailable for these days.

Fig. 2 plots the water level and the measured static deformation at two locations $(90 \mathrm{~m}$ and $172.5 \mathrm{~m})$ along vertical line NPL2 (one of three vertical lines

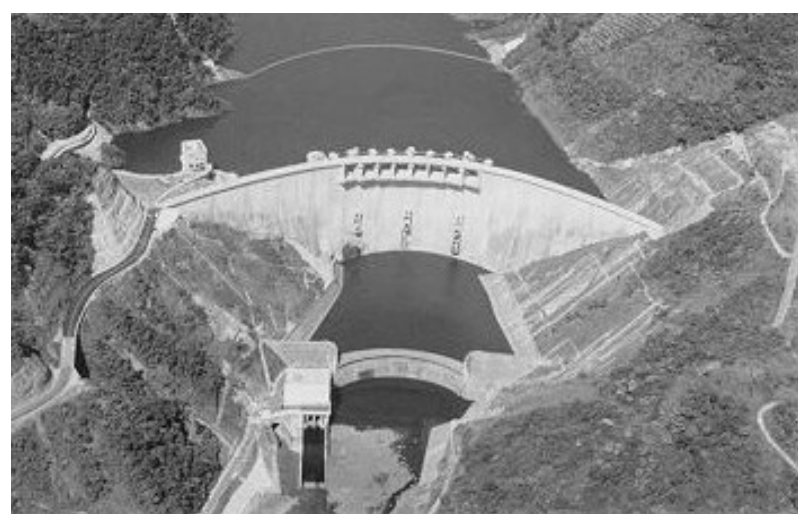

Fig. 1 Areal photo of Fei-Tsui Dam.
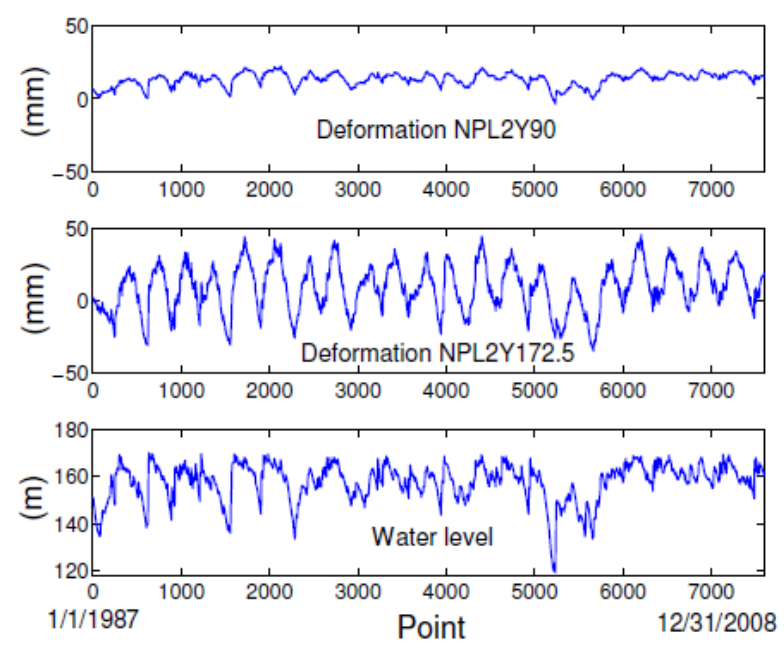

Fig. 2 Recorded water level and static radial deformations of Fei-Tsui Dam along plumb NPL2 vertical line. 
for static deformation measurement) [6]. The abscissa of Fig. 2 indicates the number of the point (from $1 / 1 / 1987$ to $12 / 31 / 2008$, a total of 7,600 data points). The magnitude of the deformation increases as the height of the measurement point increases, and it is relatively insignificant at the height of $90 \mathrm{~m}$. The signatures of the deformations at different heights are very similar to each other [11].

\section{Modeling Technique}

From a large amount of the measured data, the statistical and identification procedures have been applied to characterize the static deformation as a linear regression model [9]. This analytical model is the sum of three terms:

- the effect of hydrostatic pressure;

- the effect of temperature variation;

- the effect of time.

The model of deformation is expressed as

$$
y_{m}\left(d_{i}\right)=y_{h}\left(d_{i}\right)+y_{t}\left(d_{i}\right)+y_{d}\left(d_{i}\right)
$$

where, $y_{h}\left(d_{i}\right), y_{t}\left(d_{i}\right)$ and $y_{d}\left(d_{i}\right)$ are the effects due to hydrostatic pressure, temperature variation and time at the $i$ th measurement date $d_{i}$. The effect of hydrostatic pressure is expressed as the polynomials of water level [9]:

$$
y_{h}\left(d_{i}\right)=a_{1} h\left(d_{i}\right)+a_{2} h^{2}\left(d_{i}\right)
$$

where, $h\left(d_{i}\right)$ is the measured water level. The temperature effect is the combination of the seasonal temperature effect, expressed as a sinusoidal function with a period $c_{1}$ (close to 365 for a year), and the effect of daily temperature fluctuation as:

$$
y_{t}\left(d_{i}\right)=a_{3} t\left(d_{i}\right)+a_{4} \cos \left(\frac{2 \pi d_{i}}{c_{1}}\right)+a_{5} \sin \left(\frac{2 \pi d_{i}}{c_{1}}\right)
$$

where, $t\left(d_{i}\right)$ is the measured temperature. The time effect can be expressed as:

$$
y_{d}\left(d_{i}\right)=a_{6} d_{i}+a_{7}
$$

For any period $c_{1}$, a cost function is formed as a quadratic function of the parameters $a_{i}$ as:

$$
J=\sum_{i=n_{0}}^{n_{1}}\left[y_{a}\left(d_{i}\right)-y\left(d_{i}\right)\right]^{2}
$$

where, $y\left(d_{i}\right)$ is the measured static deformation with the chosen data prints from $n_{0}$ to $n_{1}$, and $y_{a}\left(d_{i}\right)$ is the sum of the three terms in Eqs. (2)-(4). This is a linear least-squares problem, and a unique solution of parameters $a_{i}$ can be obtained by minimizing the cost function $J$. The minimum cost function is computed as $J_{a}$, and it can be expressed as a function of parameter $c_{1}$ as $J_{a}\left(c_{1}\right)$. The problem is to find a solution of parameter $c_{1}$ to minimize the model error as:

$$
\min _{c_{1}} J_{a}\left(c_{1}\right)
$$

The MATLAB program "fminsearch", which is based on the Nelder-Mead simplex (direct search) method, is applied to get the solution of this optimization problem. The error of this identified model is computed as:

$$
y_{e}\left(d_{i}\right)=y_{m}\left(d_{i}\right)-y\left(d_{i}\right)
$$

\section{Results and Discussion}

Next, this paper will investigate the results of the application of the proposed approach to the static deformation measurements of Fei-Tsui Dam. The investigation in this paper is based on the analysis of the measured static deformation at location NPL2Y172.5 (at level $172.5 \mathrm{~m}$ ), which is always above water level as shown in Fig. 2.

\subsection{Seasonal Temperature Effect}

The previous study shows that the identified optimal period $c_{1}$ varies with the data length $n_{1}-n_{0}+$ 1 (Eq. (5)), and the measurement time interval, $n_{0} \sim n_{1}$. Next, examples are used to demonstrate the variations of the identified period and model error under two variables, the data length and the measured time. These two variables are defined as:

$$
n_{l}=n_{1}-n_{0}+1 ; \quad n_{t}=n_{0}+\frac{n_{l}}{2}-1
$$

where, $n_{t}$ represents the middle point of the chosen data for modeling. Fig. 3 shows the results of the 


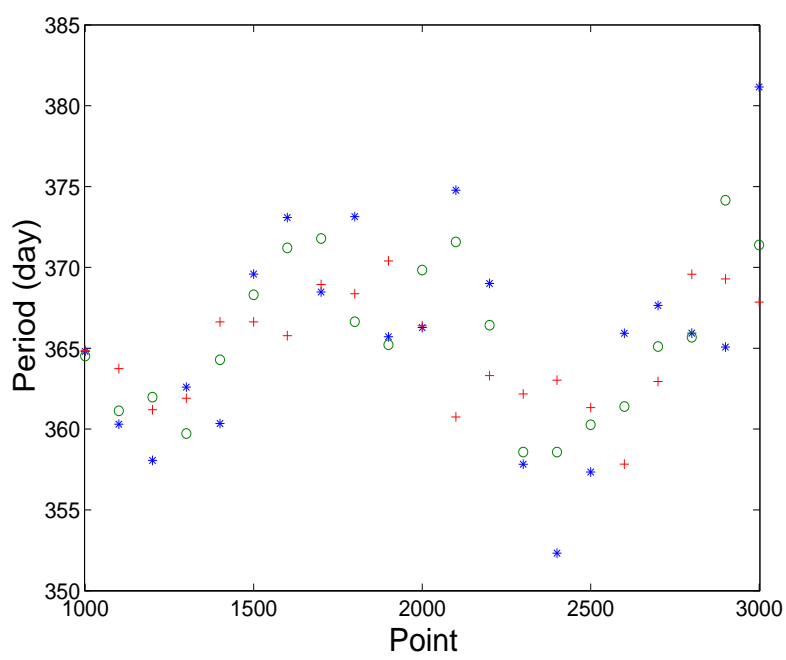

Fig. 3 Identified optimal periods $c_{1}$ of various cases $\left(*: n_{l}=600 ; 0: n_{l}=800 ;+: n_{l}=1,000\right)$.

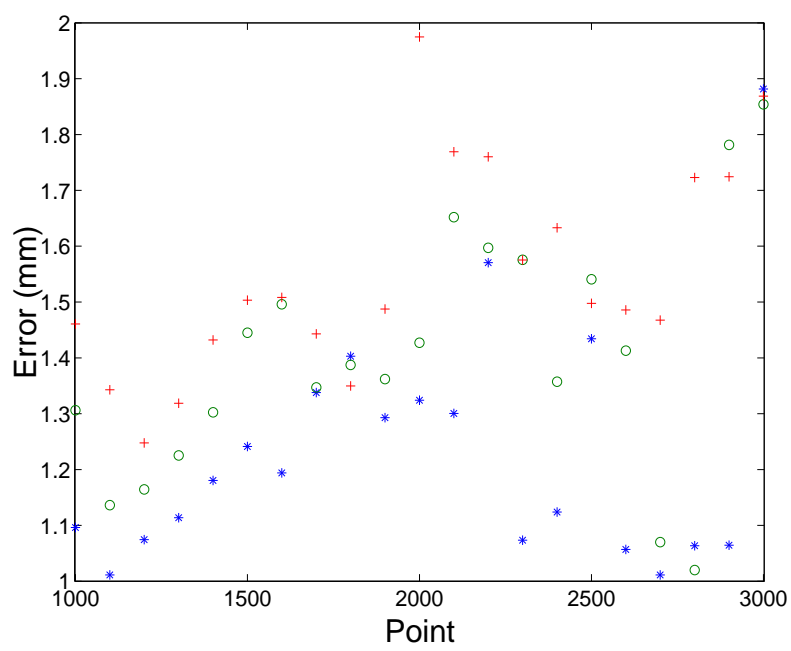

Fig. 4 Standard deviations $\sigma$ of the identified model errors of various cases $\left(*: n_{l}=600 ; 0: n_{l}=800 ;+: n_{l}=1,000\right)$.

identified optimal period $c_{1}$ for three chosen data lengths and the measured time $n_{t}$ from 1,000 to 3,000 with an increment of 100 . Fig. 4 shows the results of the standard deviation $\sigma$ of the identified model error $y_{e}$ for the cases in Fig. 3. To demonstrate the results, Table 1 lists the mean value, the maximum value, and the minimum value of the results in Figs. 3 and 4.
From the results, the following observations are noted:

(1) The range of the identified optimal period $c_{1}$ decreases as the data length $n_{l}$ increases with more data points used, as shown in Table 1 and Fig. 3. The identified period $c_{1}$ increases or decreases during a period of time $n_{t}$, for example $c_{1}$ increases from 358.6 to 374.2 as $n_{t}$ is from 2,400 to 2,900 for $n_{l}=800$;

(2) The identified model error increases as the data length $n_{l}$ increases with more data points used, as shown in Table 1 and Fig. 4. The standard deviation $\sigma$ increases or decreases during a period of time $n_{t}$, for example the standard deviation $\sigma$ of model error increases from 1.136 to 1.495 as $n_{t}$ is from 1,100 to 1,600 for $n_{l}=800$;

(3) In general, the use of more data points, larger $n_{l}$, provides an identified model with more global representation. But the use of more data points sacrifices the performance of the identified model and produces larger model error.

Next, the accuracy of the identified model is demonstrated by the case with $n_{l}=1,000$ and $n_{t}=$ 1,500. The results of this case are based on the use of Eq. (5) to analyze the measured deformation from 1,001 to 2,000 data points with various period $c_{1}$. Fig. 5 shows the standard deviation $\sigma$ of model error as a function of period $c_{1}$. The optimal solution of this case has the optimal period $c_{1}=366.63$, as shown in Figs. 3 and 5, and the standard deviation $\sigma=1.5034$, as shown in Figs. 4 and 5. Fig. 6 shows the results of the identified model with the optimal period for this case. Fig. 6a shows that the identified model well represents the experimental data. Fig. $6 \mathrm{~b}$ shows that the predicted deformation, which is estimated based on the identified model, is very close to the measured deformation.

Table 1 Results of identified models for various cases.

\begin{tabular}{lllllll}
\hline & Period $c_{1}$ & Period $c_{1}$ & Period $c_{1}$ & $\sigma$ (model error) & $\sigma$ (model error) & $\sigma$ (model error) \\
& $n_{l}=600$ & $n_{l}=800$ & $n_{l}=1,000$ & $n_{l}=600$ & $n_{l}=800$ & $n_{l}=1,000$ \\
\hline Mean & 365.686 & 365.609 & 364.902 & 1.2309 & 1.4029 & 1.5511 \\
Maximum & 381.156 & 374.153 & 370.395 & 1.8815 & 1.8540 & 1.9748 \\
Minimum & 352.322 & 358.578 & 357.837 & 1.0112 & 1.0200 & 1.2476 \\
\hline
\end{tabular}




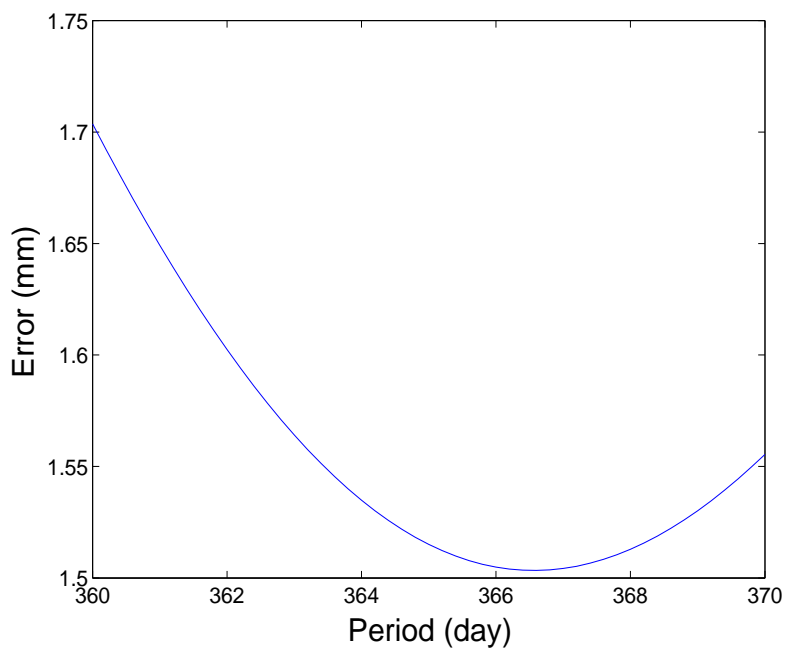

Fig. 5 Standard deviation $\sigma$ of model error as a function of period $c_{1}$ for identified models based on data from 1,001 to 2,000 points at NPL2Y172.5.

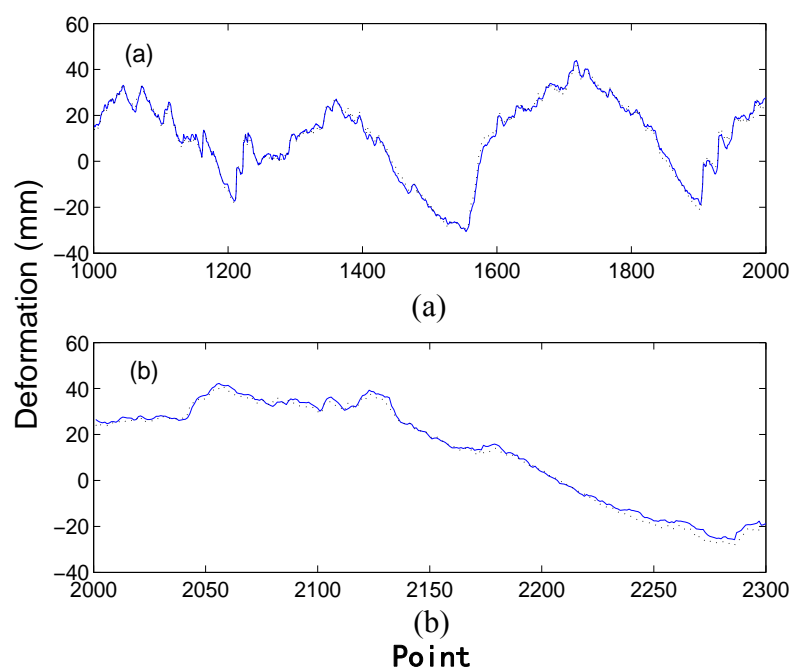

Fig. 6 Results of deformation analysis: (a) identification results; (b) prediction results (-: experimental data; $\cdots$ identified model).

\subsection{Hydrostatic Effect}

The identified parameters of the effect of hydrostatic pressure vary with environmental factors, such as drought and flooding. For investigation, the effect of the hydrostatic pressure is expressed as a function of the first order polynomial of water level instead of the second order polynomial in Eq. (2). The study is based on the identified models of the data with various length $n_{l}$ and the period $c_{1}$ from 363 to 367 with an increment of 0.4 . The results are based on the analysis of the deformation with various length $n_{l}$ in the different time intervals from the first 6,000 data points with $n_{0}$ starting from 1 with an increment of 200. The mean value of the water level for the time interval $n_{0} \sim n_{1}$, is computed as:

$$
h_{\mathrm{m}}\left(n_{t}\right)=\frac{1}{n_{1}-n_{0}+1} \sum_{i=n_{0}}^{n_{1}} h\left(d_{i}\right)
$$

Figs. 7-9 show the identified variable $a_{1}$ as a function of the mean water level $h_{\mathrm{m}}$ for the cases with $n_{l}=1,000,800$ and 600 , respectively. The line in each figure is a linear least-squares model to represent the

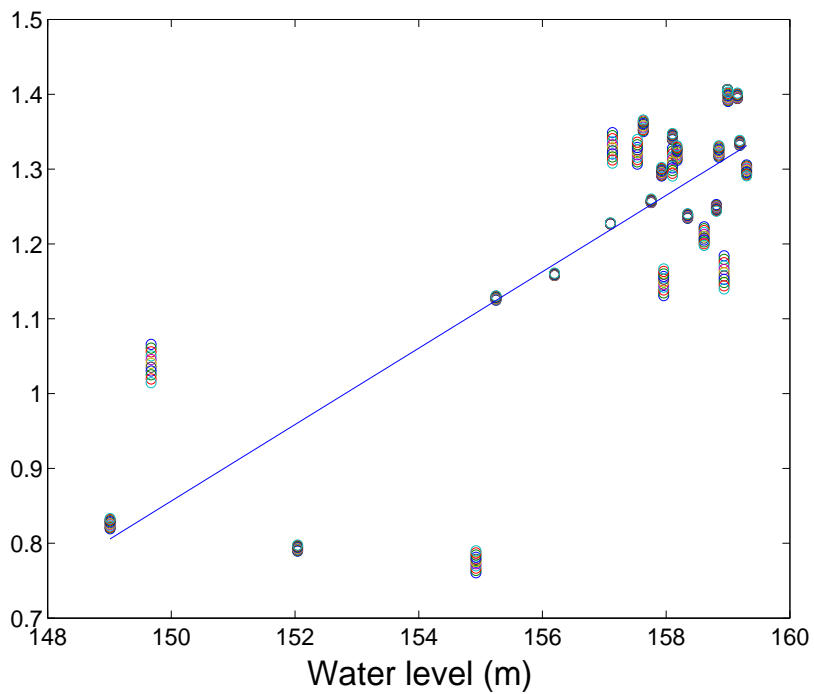

Fig. 7 Identified parameter $a_{1}$ as a function of the mean water level $h_{\mathrm{m}}$ for the cases with $n_{l}=1,000$ and $c_{1}$ from 363 to 367 .

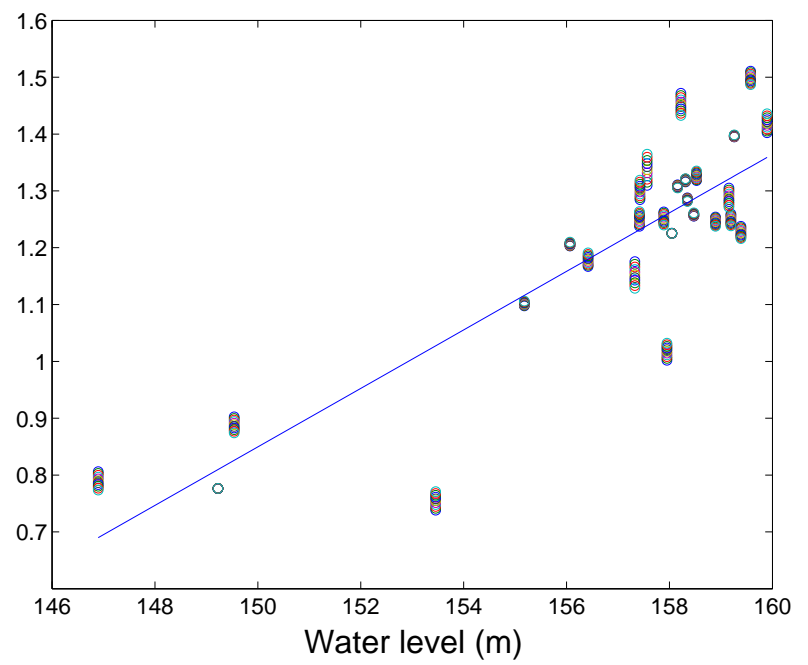

Fig. 8 Identified parameter $a_{1}$ as a function of the mean water level $h_{\mathrm{m}}$ for the cases with $n_{l}=800$ and $c_{1}$ from 363 to 367. 


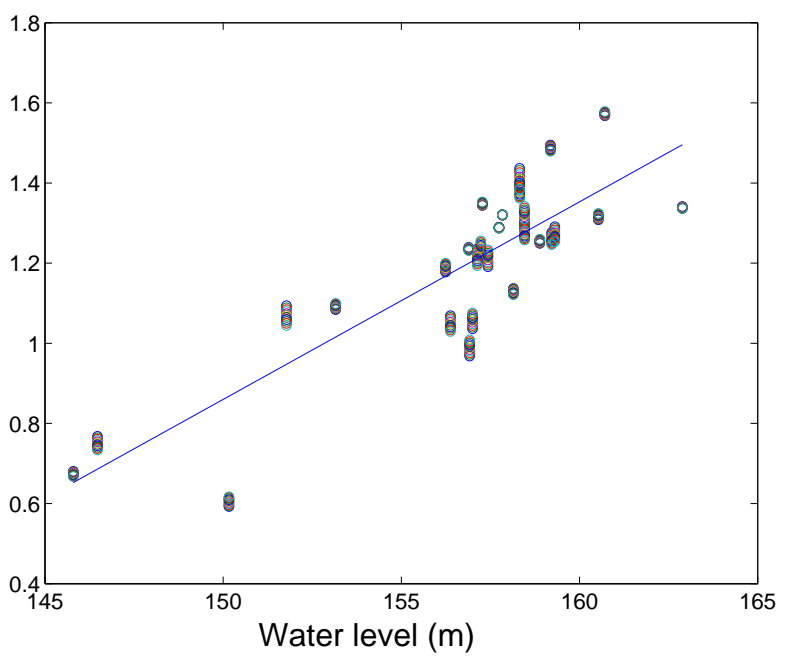

Fig. 9 Identified parameter $a_{1}$ as a function of the mean water level $h_{\mathrm{m}}$ for the cases with $n_{l}=600$ and $c_{1}$ from 363 to 367.

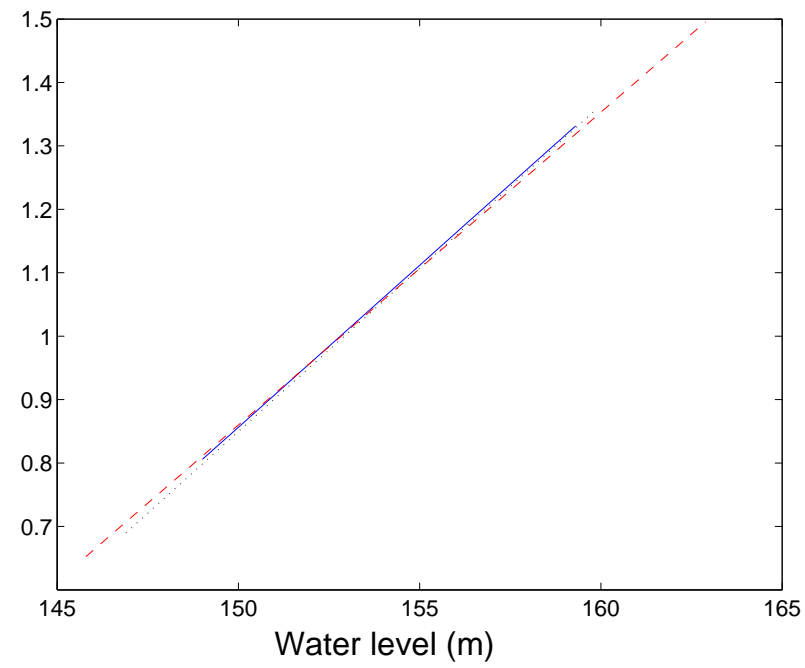

Fig. 10 Parameter $a_{1}$ as a linear function of the mean water level $h_{\mathrm{m}}:-n_{l}=1000 ; \cdots n_{l}=800 ;--n_{l}=600$.

identified parameters $a_{1}$ of various cases. Because the mean water level $h_{\mathrm{m}}$ is the average for a long period of time, more than one and a half years, the identified line in Fig. 9 relatively well represents the identified parameters $a_{1}$ of various cases with $n_{l}=600$. The model error between the line and data for the cases with $n_{l}=1,000$ in Fig. 7 is the largest due to the longest number of data points used. The results demonstrate that the identified parameter $a_{1}$ increases with water level. Fig. 10 plots the three lines in Figs. 7-9. These three lines are very close to each other, and the slopes of three lines are almost the same.

\section{Conclusions}

This paper presents an investigation of the parameter variations of the identified model of the long-term deformation of Fei-Tsui Dam. The results demonstrate that the identified period of the sinusoidal function for the seasonal temperature effect may vary significantly. The inclusion of more data points in the identified model can reduce the variation of the identified period, but it increases the model error of the identified model. The results demonstrate that the identified model well represents the experimental data and the estimated future deformation accurately represents the measured deformation. The investigation of the hydrostatic effect is based on the model with the linear effect (identified parameter $a_{1}$ ) of the measured water level on static deformation. The results demonstrate that the identified parameter $a_{1}$ increases linearly with water level, and the increased slopes are very close for the cases with various data lengths $n_{l}$. The investigation in this paper furthers the development of the reliable and effective structural health monitoring system for Fei-Tsui Dam.

\section{References}

[1] Brownjohn, J. M. W. 2007. "Structural Health Monitoring of Civil Infrastructure." Philosophical Transactions of the Royal Society A 365: 589-622.

[2] Su, J. Z., Xia, Y., Chen, L., Zhao, X., Zhang, Q. L., and $\mathrm{Xu}$, Y. L., et al. 2013. "Long-Term Structural Performance Monitoring System for the Shanghai Tower." Journal of Civil Structural Health Monitoring 3: 49-61.

[3] Fanelli, M. A. 1992. The Safety of Large Dams, Engineering Safety, edited by Blockley, D. Berkshire: McGraw-Hill.

[4] Sohn, H., Farrar, C. R., Hemez, F. M., Czarneki, J. J., Shunk, D. D., and Stinemates, D. W. 2002. A Review of Structural Health Monitoring Literature: 1996-2001. Los Alamos National Laboratory Report, LA-13976-MS.

[5] Lew, J. S. 1995. "Using Transfer Function Parameter Changes for Damage Detection of Structures." AIAA Journal 33 (11): 2189-93.

[6] Loh, C. H., Hsu, T. Y., and Chen, C. H. 2011. "Application of Advanced Statistical Methods for Extracting Long-Term Trends in Static Monitoring Data 
from an Arch Dam.” Structural Health Monitoring 10 (6): 587-601.

[7] Peeters, B., and De Roeck, G. 2001. "One-Year Monitoring of the Z24-Bridge: Environmental Effects Versus Damage Events." Earthquake Engineering and Structural Dynamics 30: 149-71.

[8] Sohn, H., Dzwonczyk, M., Straser, E. G., Kiremidjian, A. S., Law, K. H., and Meng, T. 1999. “An Experimental Study of Temperature Effect on Modal Parameters of the Alamosa Canyon Bridge." Earthquake Engineering and
Structural Dynamics 28: 879-97.

[9] De Sortis, A., and Paoliani, P. 2007. "Statistical Analysis and Structural Identification in Concrete Dam Monitoring." Eng. Struct. 29 (1): 110-20.

[10] Mata, J. 2011. "Interpretation of Concrete Dam Behaviour with Artificial Neural Network and Multiple Linear Regression Models.” Eng. Struct. 33 (3): 903-10.

[11] Lew, J. S., and Loh, C. H. 2014. "Structural Health Monitoring of an Arch Dam from Static Deformation." Journal of Civil Structural Health Monitoring 4: 245-53. 